\title{
File Conversion Application using Kivy and Tesseract - OCR
}

\author{
Swapnil More ${ }^{1}$, Rishabh Jain², Harshil Kanakia ${ }^{3}$ \\ ${ }^{1,2}$ Student. MCA Department, Sardar Patel Institute of Technology, Mumbai, India \\ ${ }^{3}$ Professor. MCA Department, Sardar Patel institute of Technology, Mumbai, India
}

Received: 21 Mar 2021; Received in revised form: 29 Apr 2021; Accepted: 16 May 2021; Available online: 31 May 2021

\begin{abstract}
In the fastest developing world education is the most important factor in all over the country. Indian Rural teachers have a great responsibility on their shoulders to educate the students without getting technological support. They face various challenges like network problems, lack of technical skills, the burden of multitasking. In this research, paper researchers try to make an application for Indian rural teachers to fight technical challenges and overcome the burden on them.
\end{abstract}

Keywords—rural teachers, mobile app, kivy technology, python for android, Tesseract-OCR.

\section{INTRODUCTION}

India ranks the second position in the population. Villages are the backbone of India in villages less importance is given to education to encourage people to educate their children for this Indian government introduced various schemes and projects for the students. they look at their health and nutrition for their mental growth. For this Indian government introduced a mid-day meal scheme to feed every student they give their responsibility to teacher who work in rural area . but due to this teachers who work in rural areas get lots of burden of work in challenging situations. Teachers face lots of problems like having to maintain the record of each and every student and keep track of their progress . maintain this data and make a record of every student then to submit this record to the government office. most of the time user stay in no network zone so this app ensure to work smoothly in offline mode also.In the next section of the paper, researchers discuss and try to develop a mobile application for teachers who face the loss of challenge to manage student records with the help of kivy technology and python for android which able to work online as well as offline without getting any disturbance.

\section{LITERATURE REVIEW}

kivy technology is kind of new technology to the world which world with the advantage of python programming

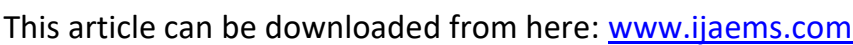

(C)2021 The Author(s). Published by Infogain Publication.

This work is licensed under a Creative Commons Attribution 4.0 License. http://creativecommons.org/licenses/by/4.0/ language, kivy is evolution of PYMT (Hansen et. al 2009) project[12] . In their paper, "Developing App for Android and Other Platforms with Kivy and Python" Andreas Schreiber give a demonstration of Kivy- based application and sum-maries Kivy framework[11]

kivy is most well known android supporting python toolkit has been since around 1012. kivy dose not specifically designed for mobile support but it focus on cross platform and supporting novel user interface this properties made it support android, IOS as well as desktop use[8]. it draw its GUI using openGL which working essentially same at all platform.kivy android support originally based on Renpy's Android build tool. it help to Create multi touch user interface and python UI framework for rapid development of mobile application[6] . 


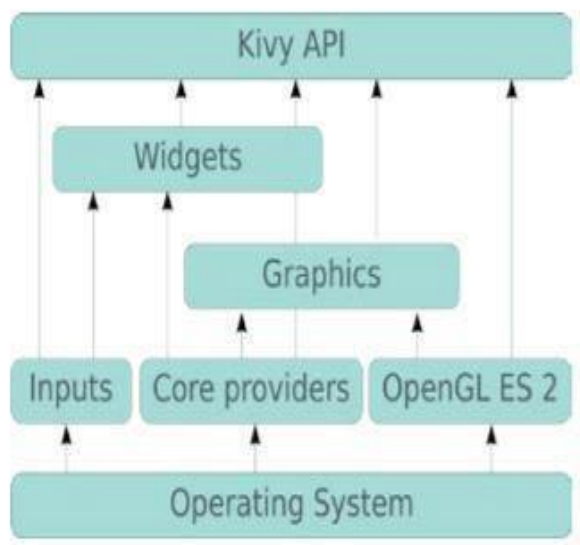

Fig 1. general architecture of Kivy [2] .

\section{METHODOLOGY}

researchers use secondary data and previous studies, research articles. researchers use observation methods to study challenges that are faced by Indian rural teachers. in order to create system that able to scan and classifies data and manages the record. In our study, we tried to create a mobile application which able to scan and upload and maintain a record of students

The proposed methodology involves :

1. Python Programming Language :-

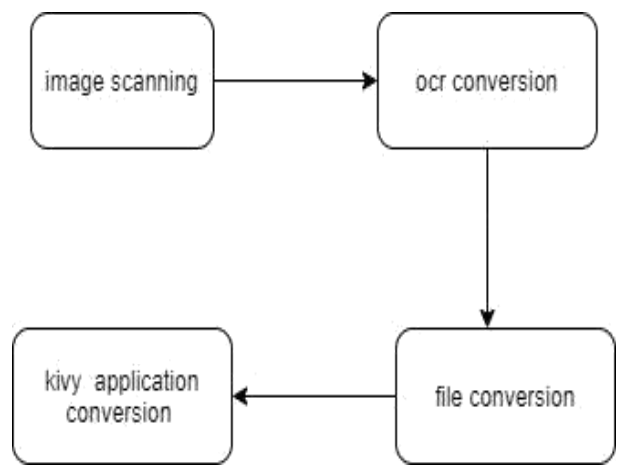

Fig 2. General Methodology

To make the Model and Write the algorithm to implement the File conversion[9].

\section{Kivy technology:-}

We use the Kivy because its framework which support the python language and its very easy to integrate with python library[2][11].

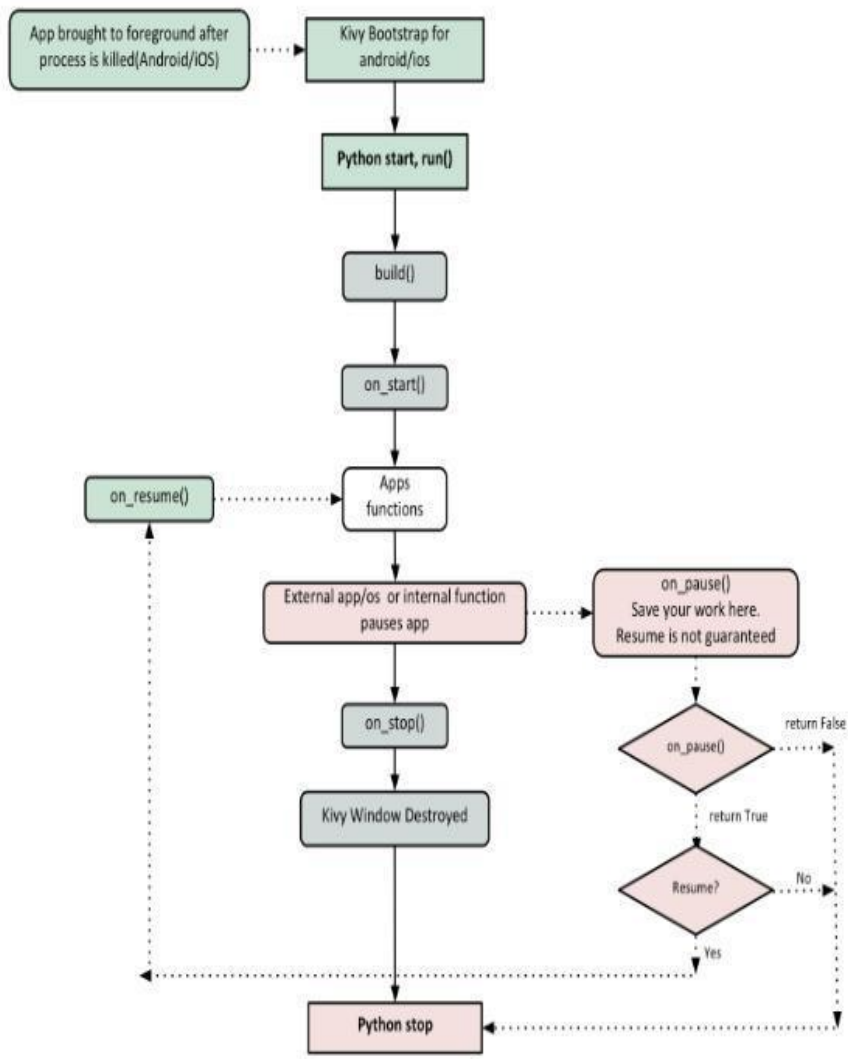

Fig 3. Kivy application life cycle .

\section{A. image Scanning}

The research aim to help teachers to minimize paperwork by scanning.the data collection by scanning implement as follows:- we provide image which present in gallery or click new image using camera researchers use:- opencv-contrib-python to get acces to extra modules scikit-image for handling image processing stuff

numpy to handle matrix

imutils to make image processing easier

we convert image form colour to gray scale and then applied bilateral filter for smoothing image

\section{B. Optical Character Recognition}

conversion of text into digital format where camera cap-ture photograph and OCR bring them into document format Python tesseract is optical character recognition tool which help to convert chararcters to digital format[4] , tesseract has implemented long short term memory based recognition engine which makes things more faster so researchers took pytesseract for imprt and Scan image[3]. the json responce the ocr image function processimage().json . Aim of Tesseract was to recognizes white on black which help to analysis and recognizes a particular character[10]. 


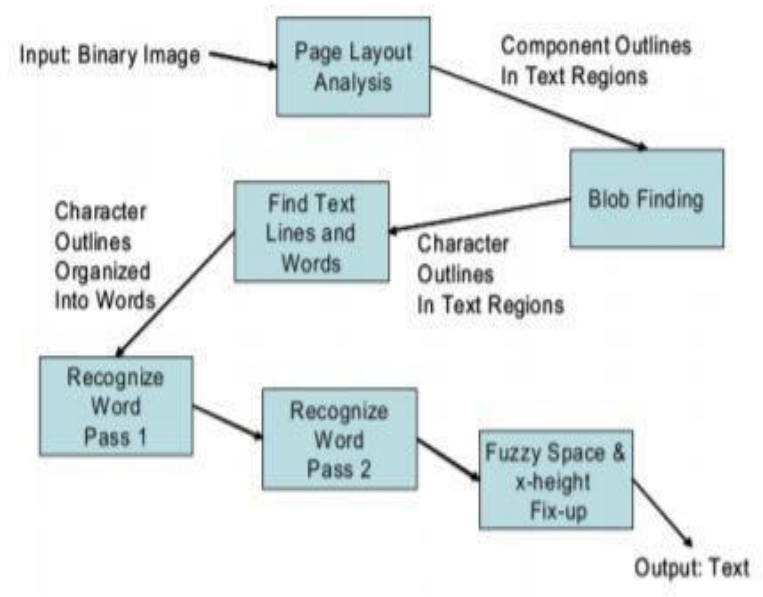

Fig 4. Block diagram of Tesseract

\section{Data cleaning And File conversion with help of NLP}

The data cleaning process get more complex when data come from some heterogeneous source, these problem has been solved by data cleaning and data transformation, data cleaning modules such as removing columns with less data removing unnecessary rows identifying some valuing and feeling it is done by Python code with the help of machine learning algorithm, most of the data got missing after cleaning then to overcome this used pyspellchecker, Text-blob and Auto-correct[1], this all are open source packages that allow to correct and check spelling, meanwhile by using Text-blob allows us to use custom database it provide simple api for divining into natural language processing(NLP) job

D. kivy application development

We take here a simple example of a minimal application[5][7] :

\section{import kivy

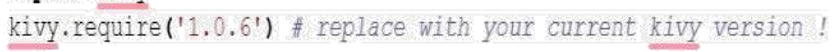

\section{from kivy. app import App \\ from kivy.dix.label import tabel}

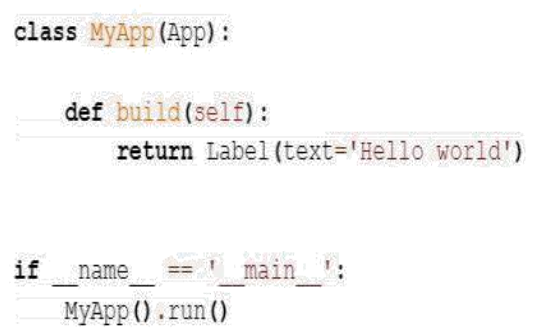

Fig 5. Sample App

This article can be downloaded from here: www.ijaems.com

\section{RESULTS}

The first test done by developers by using their application, they run test on image which contain some text . the appli-cation identify the document and as output create document from the scan image

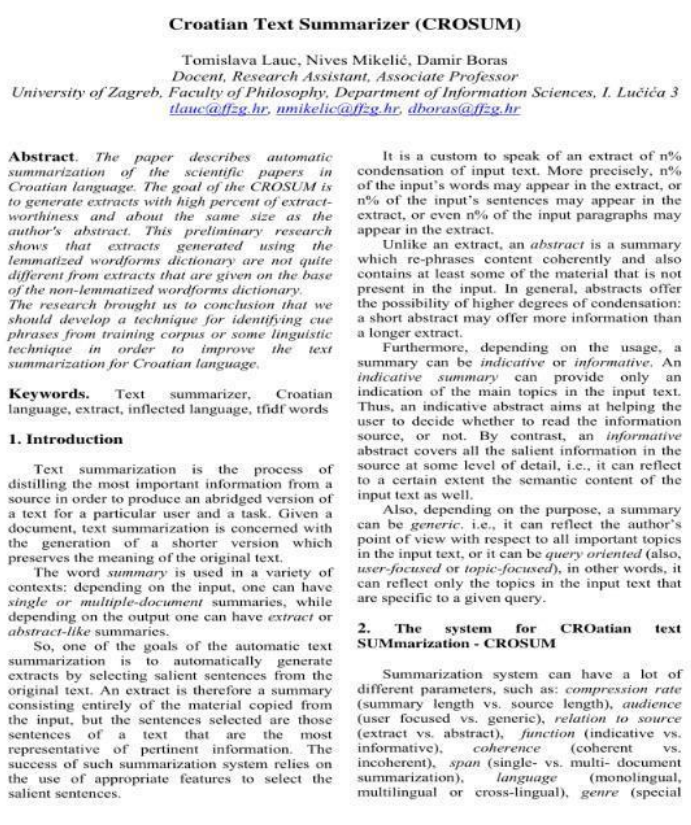

Fig 6. Generated Document

\section{CONCLUSION}

In this paper, developer tried to provide some information about kivy technology for building an android application for creating and maintaining a record. after application develop-ment developer were able to conduct the following:-

click the image from mobile

Scan and extract the data from image using character Recognition

Generate document and create record of that document Uploaded record

Previously uploaded data is reusable for mentoring and recalling

Offline access of previously uploaded data

\section{FUTURES SCOPE}

This Work on the application can be extended upon how it impacts in day to day life as well as with some recommen-dations. On the basis of requirements and technical changes, future changes will be done. the project 
can be updated in near future as requirement for the same arises some of the fallowing are the future scope for it

Document conversation without any format limitation . For getting more accuracy AI and ML implementation work is required.

Creation for master database so it can be used for further requirement.

Changes according to Organization requirement.

\section{ACKNOWLEDGMENT}

This Paper and research supported by Sardar Patel Institute Of Technology. We thank Prof. Harshil kanakia for comments that greatly improve the menu-script also we thank our faculty who provided insight and expertise that greatly assisted the research.

\section{REFERENCES}

[1] A SPELL CHECKER IN PYTHON. URL: https : / / theautomatic . net / 2019/12/10/3 - packages - to build - a-spell-checker-in-python/.

[2] Brno autum Bc. Ond•rej Chrastina. "Cross platform Development of Smartphone application with the kivy framework". In: 2015.

[3] Build Google's Tesseract and OpenCV. URL: https : / / www. analyticsvidhya . com / blog / 2020 / 05 / build your-own-ocr-google-tesseract-opencv/.

[4] Image to text conversion. URL: http://ijasc.ascons.org/digital-library/15866.

[5] Kivy Basics. URL: https : / / kivy. org / doc / stable / guide / basic.html.

[6] Kivy Cross-Platform Applications. URL: https : / / towardsdatascience . com / python - for - android - start building - kivy - cross - platform - applications 6cf867d44612.

[7] Kivy Python Framework. URL: https://realpython.com/ mobile-app-kivy-python/.

[8] Oleksander Lobunets Mathieu Virbel ThomasHansen. "Kivy - Framework For Rapid Creation ofInnovative User Interface". In:

[9] Python Modules. URL: https : / / docs . python . org / 3 / installing/index.html.

[10] Vishal Thengne Sahil Thakare Ajay Kamble and U.R. Kamble. "Document Segmentation and Language Translation Using Tesseract-OCR". In: 2018 IEEE 13th International Conference on Industrial and Information Systems (ICIIS) (2018). DOI: 10.1109/ICIINFS.2018. 8721372.

[11] Andreas Schreibe. "Developing App for Androidand Other Platforms with Kivy and Python”. In: 2013.

[12] Alexander Taylor. "An actual history of Python on Android”. In: May 2020. 